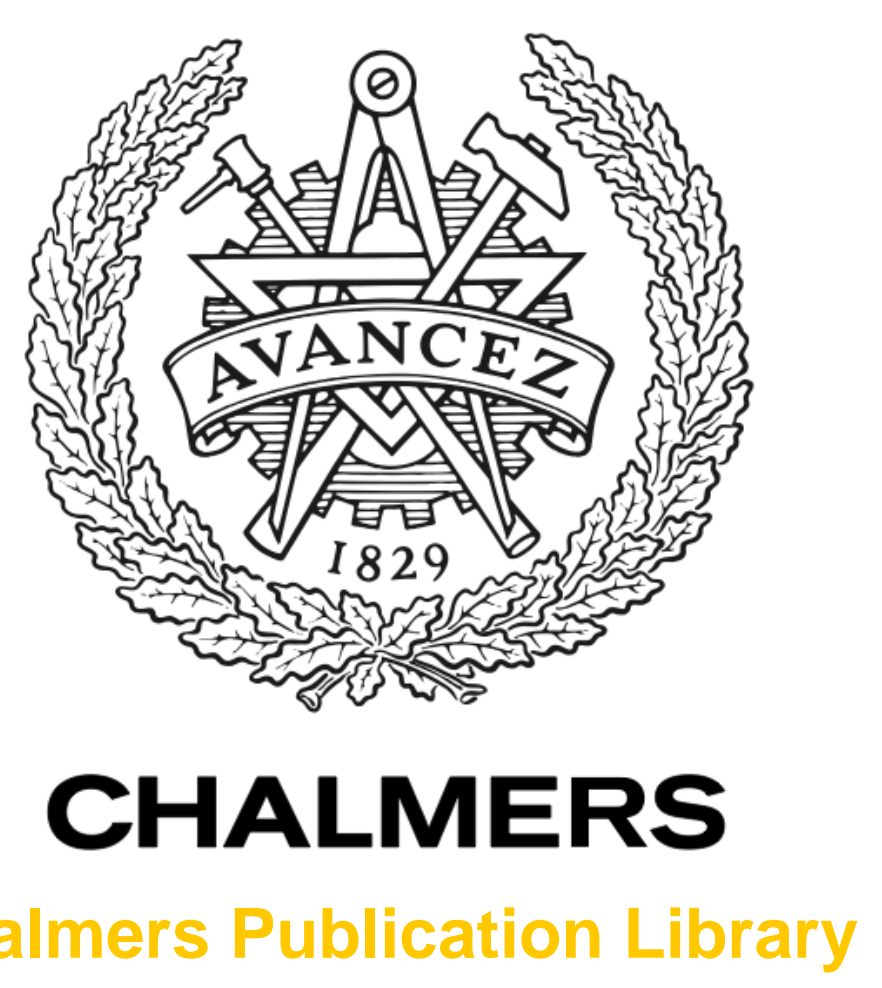

Chalmers Publication Library

\title{
Power Control for Broadcast V2V Communications with Adjacent Carrier Interference Effects
}

This document has been downloaded from Chalmers Publication Library (CPL). It is the author's version of a work that was accepted for publication in:

IEEE International Conference on Communication (ICC) 2016

Citation for the published paper:

Hisham, A. ; Wanlu, S. ; Ström, E. et al. (2016) "Power Control for Broadcast V2V

Communications with Adjacent Carrier Interference Effects". IEEE International Conference

on Communication (ICC) 2016

Downloaded from: http://publications.lib.chalmers.se/publication/237539

Notice: Changes introduced as a result of publishing processes such as copy-editing and formatting may not be reflected in this document. For a definitive version of this work, please refer to the published source. Please note that access to the published version might require a subscription. 


\title{
Power Control for Broadcast V2V Communications with Adjacent Carrier Interference Effects
}

\author{
Anver Hisham, Wanlu Sun, Erik G. Ström, and Fredrik Brännström \\ Department of Signals and Systems, Chalmers University of Technology, Gothenburg, Sweden \\ \{anver, wanlu, erik.strom, fredrik.brannstrom\}@chalmers.se
}

\begin{abstract}
This paper investigates the power control problem for broadcast vehicular communications in the presence of adjacent channel interference (ACI). First, we study the impact of ACI on vehicle-to-vehicle ( $\mathrm{V} 2 \mathrm{~V})$ communications and conclude that the ACI indeed significantly affects the reliability of $\mathrm{V2V}$ links. Second, we formulate a power control optimization problem for vehicles to reduce the negative influence of ACI, which is shown to be NP-hard. Furthermore, we propose two power control schemes where the first one solves the formulated problem by a branch and bound method and the second one considers a heuristic algorithm with much reduced complexity. Finally, simulations are presented which illustrate the necessity of power control when ACI exists and also show promising performance of the proposed algorithms.
\end{abstract}

\section{INTRODUCTION}

Intelligent transportation systems have been investigated for many years with the aim of enabling various applications to improve traffic safety, efficiency, and comfort. Vehicle-tovehicle $(\mathrm{V} 2 \mathrm{~V})$ communication, i.e., when vehicles in relative close proximity exchange data, is an enabler for cooperative traffic safety and traffic efficiency applications. Due to the safety-critical nature of the application, reliability requirements are typically very high.

Compared to conventional cellular communication, the V2V network has two novel features. First, the V2V network usually relies on broadcast protocols to disseminate safety-related messages. Second, V2V communications often come with a stringent requirement on reliability, which can be achieved if the signal to interference and noise ratio (SINR) exceeds a certain threshold [1]. To attain a high reliability, we can allocate non-overlapping resources [2], e.g., separate resource blocks (RBs) in frequency domain, to different vehicular user equipments (VUEs) for their transmissions. This way, cochannel interference (CCI) is avoided.

In practice, however, if two transmitters operate on two channel bands close to each other in frequency domain, energy from one transmitter will spill over into the frequency band of the other transmitter. This interference is termed adjacent channel interference (ACI) [3]. The ACI is mainly due to two causes. First, since practical RF filters require a rolloff factor, they cannot eliminate out of band transmission. Second, nonlinearities at the transmitter and receiver cause the transmitted spectrum to spread beyond what was intended. An example of ACI is illustrated in Fig. 1, where the receiver $j$ is decoding signals from the two transmitters $i$ and $k$ on different RBs. Take the received signal from transmitter $i$ as an example. Although transmitter $k$ is using a different RB,

The research was funded by the Swedish Governmental Agency for Innovation Systems (VINNOVA), FFI - Strategic Vehicle Research and Innovation, under Grant No. 2014-01387.
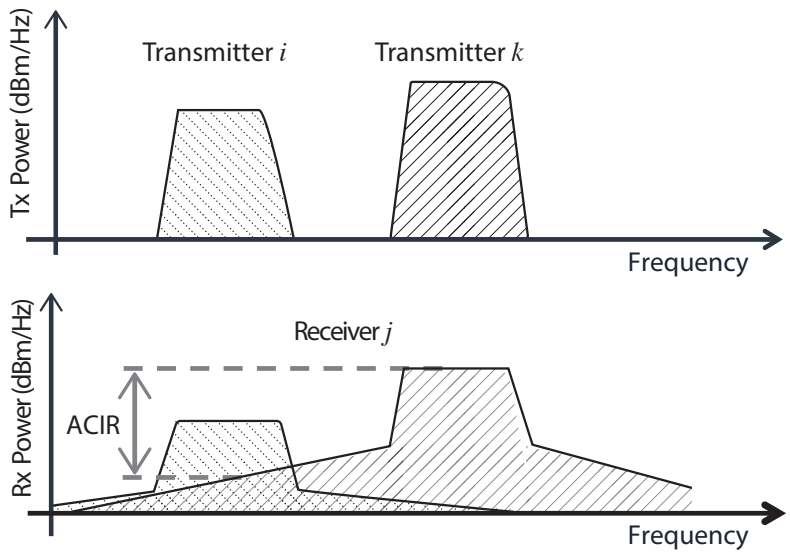

Fig. 1. Adjacent Channel Interference

the performance of the receiver when decoding the signal from transmitter $i$ is limited by ACI incurred from transmitter $k$. A parameter named adjacent channel interference ratio (ACIR) is widely used to measure the ACI [4, pp. 370-371]. As shown in Fig. 1, ACIR is defined as the ratio between the in-band received power from transmitter $k$ to the leakage power from transmitter $k$ 's signal to the RB allocated to transmitter $i$.

In the field of power control for vehicular networks, most existing literature consider the shared channel access for VUEs, i.e., following the setup in the $802.11 \mathrm{p}$ standard. In this case, CCI becomes the most harmful effect on the reliability of $\mathrm{V} 2 \mathrm{~V}$ communications. Hence, a key design issue is to tackle the CCI and thus decrease message congestion. For this purpose, various power control protocols have been proposed, see [5]-[7] for some examples. Most of them, however, adopt simple power adjustment strategies based on an estimated traffic load. This way, reliability performance is usually unsatisfactory. Moreover, a related research area is power control in multicast scenario, where more sophisticated algorithms have been devised to improve the performance at the intended receivers [8], [9].

On the other hand, due to the strict reliability requirement of V2V links, orthogonal channel access has been recognized as a promising candidate to support vehicular communications. In particular, using the Long Term Evolution (LTE) standard, separate RBs can be allocated to different VUEs for their transmissions [2], [10], [11]. In this case, CCI can be eliminated and thus, ideally, VUEs will not interfere each other. However, as explained above, ACI does exist in reality. Whether or not ACI will have a serious negative influence to the V2V 
communications, is still an open question in the literature.

In this work, we study the effect of ACI on frequencymultiplexed V2V all-to-all broadcast communication (i.e., when all VUEs are both transmitters and receivers). After some investigations, we come to the conclusion that the ACI indeed plays a role and cannot be neglected. To reduce the harmful influence of ACI, both power control and efficient RB scheduling can be used. In this paper, we have only considered power control schemes. To this end, we first formulate an optimization problem for power control of VUEs, with the objective of maximizing the number of successful V2V links in Section II-C. The formulated problem is shown to be NPhard. Moreover, we propose two power control algorithms to approximately solve the optimization in Section III-A and Section III-B. The first one achieves a near-optimal solution by the branch and bound method and the second one is a heuristic algorithm with much reduced complexity. Finally, simulations show promising results for the proposed schemes.

\section{System Model And Problem Formulation}

\section{A. System Model}

We consider a network consisting of $N$ VUEs distributed after each other on a one-lane road. The VUEs are numbered as per location index in the convoy as illustrated in Fig. 2. Assume that each VUE broadcast safety messages to neighbouring VUEs within an intended transmission range. We denote by $\mathcal{A}_{i}$ the set of the intended receivers for $i^{\text {th }}$ VUE. Let $h_{i, j}$ be the average channel power gain from $i^{\text {th }}$ VUE to $j^{\text {th }}$ VUE. Hence, $h_{i, j}$ takes into account the pathloss and large-scale fading between $i^{\text {th }}$ VUE and $j^{\text {th }}$ VUE. Let $g_{k, i, j}$ be the average channel power gain of the interference from $k^{\text {th }}$ VUE to the communication link from $i^{\text {th }}$ VUE to $j^{\text {th }}$ VUE. Clearly, the value of $g_{k, i, j}$ depends on both the physical channel value $h_{k, j}$ and the RB scheduling result for $k^{\text {th }}$ VUE and $i^{\text {th }}$ VUE, since the latter possibly brings ACI. Following the ACIR model given in [12], we have

$$
g_{k, i, j}=h_{k, j} / \operatorname{acir}\left(\Gamma_{k}-\Gamma_{i}\right)
$$

where $\Gamma_{k}$ and $\Gamma_{i}$ are the RB indices allocated to $k^{\text {th }}$ VUE and $i^{\text {th }}$ VUE respectively. The $\operatorname{acir}(x)$ denotes ACIR between two $\mathrm{RBs}$ separated by $x \mathrm{RBs}$ is given as [12]

$$
\operatorname{acir}(x)= \begin{cases}1, & x=0 \\ 10^{3}, & 1 \leq|x| \leq 4 \\ 2 \cdot 10^{4}, & \text { otherwise }\end{cases}
$$

The scenario $x=0$ in the above equation implies that VUEs are allocated with same $\mathrm{RB}$, in which case the interference is CCI instead of ACI. In our study, this scenario never happens, since no RB is used by more than one VUE.

Under the assumption of non-overlapping RB allocation for VUEs, where the $i^{\text {th }}$ VUE broadcasts on RB $i$, the received SINR $\gamma_{i, j}$ of the link from $i^{\text {th }}$ VUE to $j^{\text {th }}$ VUE is computed as

$$
\gamma_{i, j}=\frac{P_{i} h_{i, j}}{\sigma^{2}+\sum_{\substack{k=1,1 \\ k \neq i}}^{N} P_{k} g_{k, i, j}}
$$

where $P_{i}$ is the transmit power of $i^{\text {th }}$ VUE and $\sigma^{2}$ denotes the noise power. The link between $i^{\text {th }}$ VUE and $j^{\text {th }}$ VUE is

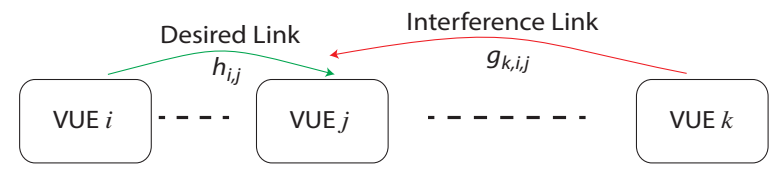

Fig. 2. System model

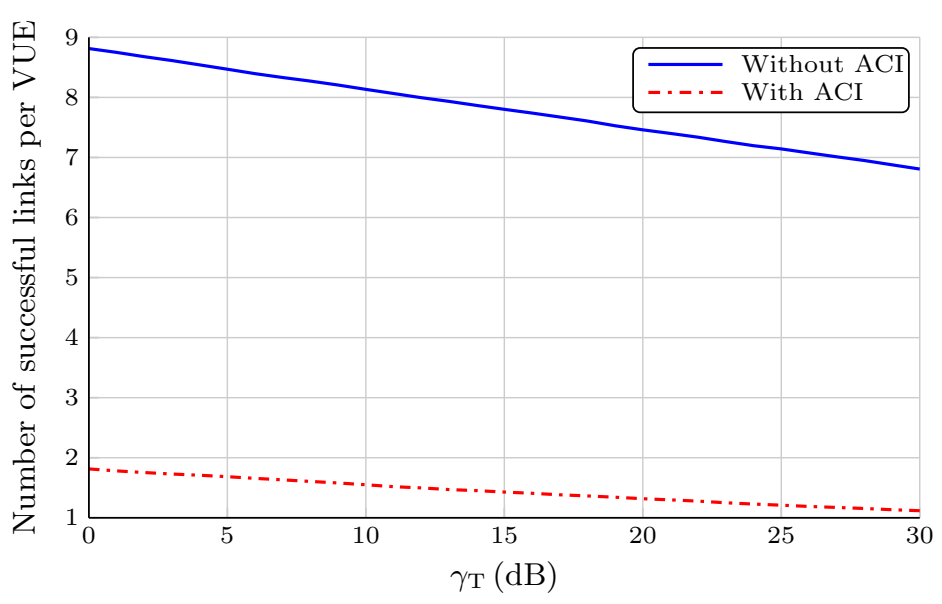

Fig. 3. Impact of ACI $(N=20)$

considered successful if $\gamma_{i, j} \geq \gamma_{\mathrm{T}}$, where the SINR threshold, $\gamma_{\mathrm{T}}$, is assumed to be known. In general, $\gamma_{\mathrm{T}}$ depends on several factors, including the packet size and the reliability requirement [1].

\section{B. Some Observations about ACI}

Now we investigate the impact of ACI on V2V communications in the absence of CCI. The channel model and VUE position distribution are as explained in Section IV. Here we assume that all VUEs broadcast with the same power $P_{\max }$.

Recall that a successful link is defined as a link with $\gamma_{i, j} \geq$ $\gamma_{\mathrm{T}}$. With respect to varied $\gamma_{\mathrm{T}}$, the average number of successful links per VUE is plotted in Fig. 3, where we see a significant gap between the cases with and without ACI. Hence, it can be concluded that the performance of $\mathrm{V} 2 \mathrm{~V}$ communication is indeed highly impacted by ACI.

\section{Problem Formulation}

In reality, due to the link quality and due to the interference from adjacent RBs, reliable transmission from the $i^{\text {th }}$ VUE cannot in general be guaranteed to all of its intended receivers $\mathcal{A}_{i}$. We define binary variables $x_{i, j}$, for all $i=1, \ldots, N$ and $j \in \mathcal{A}_{i}$, to indicate the success of the link from VUE $i$ to VUE $j$, i.e.,

$$
x_{i, j} \triangleq \begin{cases}1, & \gamma_{i, j} \geq \gamma_{\mathrm{T}} \\ 0, & \text { otherwise }\end{cases}
$$

To achieve a more reliable broadcast of safety messages, we set our aim to maximize the number of successful links by adjusting the transmit power per VUE. Besides, when different power allocation results yield the same number of successful links, we probably prefer a lower transmit power. In this way, the power control problem for broadcasting VUEs is formulated as follows. 


$$
\max _{x_{i, j}, P_{i}}\left(\sum_{i=1}^{N} \sum_{j \in \mathcal{A}_{i}} x_{i, j}-\beta \sum_{i=1}^{N} P_{i}\right)
$$

subject to:

$$
\begin{aligned}
& x_{i, j} \in\{0,1\} \quad \forall i, j \\
& 0 \leq P_{i} \leq P_{\max }^{N} \quad \forall i \\
& P_{i} h_{i, j}-\gamma_{\mathrm{T}} \sum_{\substack{k=1, k \neq i}}^{N} P_{k} g_{k, i, j} \geq \gamma_{\mathrm{T}} \sigma^{2}-\eta\left(1-x_{i, j}\right) \quad \forall i, j
\end{aligned}
$$

where $\beta$ is the weight of the total power consumption in the objective, which is set to be a small nonnegative number such that the sum power will not affect our major goal of maximizing the number of successful links. For example, we can choose $\beta=1 /\left(N P_{\max }\right)$. Besides, constraint (5c) states the max transmit power for each VUE. The inequality in (5d) ensures the SINR constraint is satisfied for the successful links, where $\eta$ is a sufficiently large positive number. Specifically, constraint (5d) forces $\gamma_{i, j} \geq \gamma_{\mathrm{T}}$ if the link from $i$ to $j$ is considered successful, i.e., $x_{i, j}=1$; otherwise, if $x_{i, j}=0$, (5d) becomes redundant due to the large value of $\eta$. Under the reasonable assumption that $g_{k, i, j}<1$, then $\eta=\gamma_{\mathrm{T}}\left(\sigma^{2}+N P_{\max }\right)$ is sufficiently large.

Lemma 1: The problem formulated in (5) is NP-hard.

Proof: By setting cardinality of $\mathcal{A}_{i}=1$ for all $i$ and $\beta=0$, problem (5) becomes a traditional problem of finding the maximum number of transmitters that can be supported at a given SINR target for a unicast system. The latter has been shown in [13] to be NP-hard. Thus, the problem (5) is also NP-hard.

\section{Power Control Algorithm}

As investigated above, ACI has indeed a significant effect on V2V communications and cannot be neglected. Therefore, we will exploit a power control strategy to reduce the negative influence of ACI, i.e., solve problem (5). To this end, we will propose two power control algorithms in the following subsections.

\section{A. Near-Optimal Power Control by Branch and Bound}

Problem (5) is essentially a mixed integer linear programs (MILP), which can be solved by branch and bound (BnB) algorithms [14] using the following divide and conquer strategy. The feasible set of the original problem can be divided into two subsets where one binary variable, e.g., an $x_{i, j}$ in (5), is fixed to either 0 or 1 while other variables are unchanged. This way, the associated MILP subproblem has one variable fixed or eliminated. By solving linear relaxations of these subproblems, the lower and upper bound of the objective of each subproblem are obtained [14]. Then the subsets are further split into smaller subsets using the same idea and the corresponding linear relaxations are again solved for more accurate lower and upper bounds. During this process, we update the global lower and upper bounds, and prune the unpromising subsets whose upper bounds are smaller than the best-known global lower bound. The procedure is carried out iteratively until that the gap between the global lower and upper bounds converges to a preset stop criterion.

Branch and bound algorithms are attractive in that they maintain a provable upper and lower bound on the global optimum [14]. However, due to the NP-hardness of problem (5), branch and bound algorithms involve a number of linear optimizations which, in the worst case, is believed to be exponential in the size of binary variables.

\section{B. Heuristic Power Control Algorithm}

The exponentially increased worst-case complexity for obtaining the near-optimal solution by the $\mathrm{BnB}$ method, is problematic in practice, especially for a large network. Hence, we propose a heuristic power control algorithm with much reduced complexity. For notational convenience, we define the union of all the intended links as

$$
\mathcal{A}=\left\{(i, j): 1 \leq i \leq N, j \in \mathcal{A}_{i}\right\} .
$$

Clearly, if we have $\gamma_{i, j} \geq \gamma_{\mathrm{T}}$ for all $(i, j) \in \mathcal{A}$, the remaining problem is quite simple since problem (5) will reduce to a pure linear programming problem. However, the difficulty lies in what links to include when not all of them are declared successful. To address this problem, we propose an iterative power control algorithm, inspired by [8] and described in Fig. 4. The two main features of the proposed scheme are 1) each VUE $i$ iteratively updates its transmit power $P_{i}$ in a distributed manner, based on the SINR measurement reported back from its intended receivers and 2) each communication link $(i, j)$ maintains the counter $C(i, j)$ such that this link is declared unsuccessful only when $C(i, j)$ exceeds a threshold $C_{\mathrm{T}}$.

Next we explain each step in Fig. 4 in detail. Firstly, each VUE $i$ initializes its transmit power $P_{i}$ to $P_{\text {init }}$ and a counter $C(i, j)=0$ for all $j \in \mathcal{A}_{i}$. $P_{\text {init }}$ is chosen to be an arbitrarily small value compared to $P_{\max }$, so that the computed required power values are less than $P_{\max }$ in the first iteration of the algorithm. Also, we denote by $\mathcal{S}$ the set of currently active links and initialize $\mathcal{S}=\mathcal{A}$. By an active link here we mean a communication link that is not declared unsuccessful yet. Then, the iteration starts by each receiver of the links in $\mathcal{S}$ measuring $\gamma_{i, j}$. Naturally, if $\gamma_{i, j} \geq \gamma_{\mathrm{T}}$ for all $(i, j) \in \mathcal{S}$, we have solved the problem (5), since all the intended links have SINR above $\gamma_{\mathrm{T}}$. Otherwise, we compute a required transmit power $\tilde{P}_{i, j}$ for all $(i, j) \in \mathcal{S}$ such that these links reach exactly $\gamma_{\mathrm{T}}$ under the assumption that the interference remain constant and equal to the currently measured interference. Intuitively, the link $(i, j)$ is unsuccessful if $\tilde{P}_{i, j}>P_{\max }$. However, it is not necessarily true since the links are coupled and the removal of one link may affect the success of another link. Hence, we will not immediately remove the link $(i, j)$ even when $\tilde{P}_{i, j}>P_{\max }$. Instead, we define $\mathcal{W} \triangleq\left\{(i, j) \in \mathcal{S} \mid \tilde{P}_{i, j}>P_{\max }\right\}$ as the set of candidate links to be removed and increase the counter $C(i, j)=C(i, j)+1$ for all $(i, j) \in \mathcal{W}$. The introduction of the counter $C(i, j)$ is interpreted as a deferred removal strategy since the link $(i, j)$ is removed from $\mathcal{S}$ only when $C(i, j)>C_{\mathrm{T}}$. Furthermore, the transmit power is updated as $P_{i}=\max _{j:(i, j) \in \mathcal{S} \backslash \mathcal{W}}\left(\tilde{P}_{i, j}\right)$ for all $i$. Then the next iteration starts based on the updated $P_{i}$ values and goes through the 


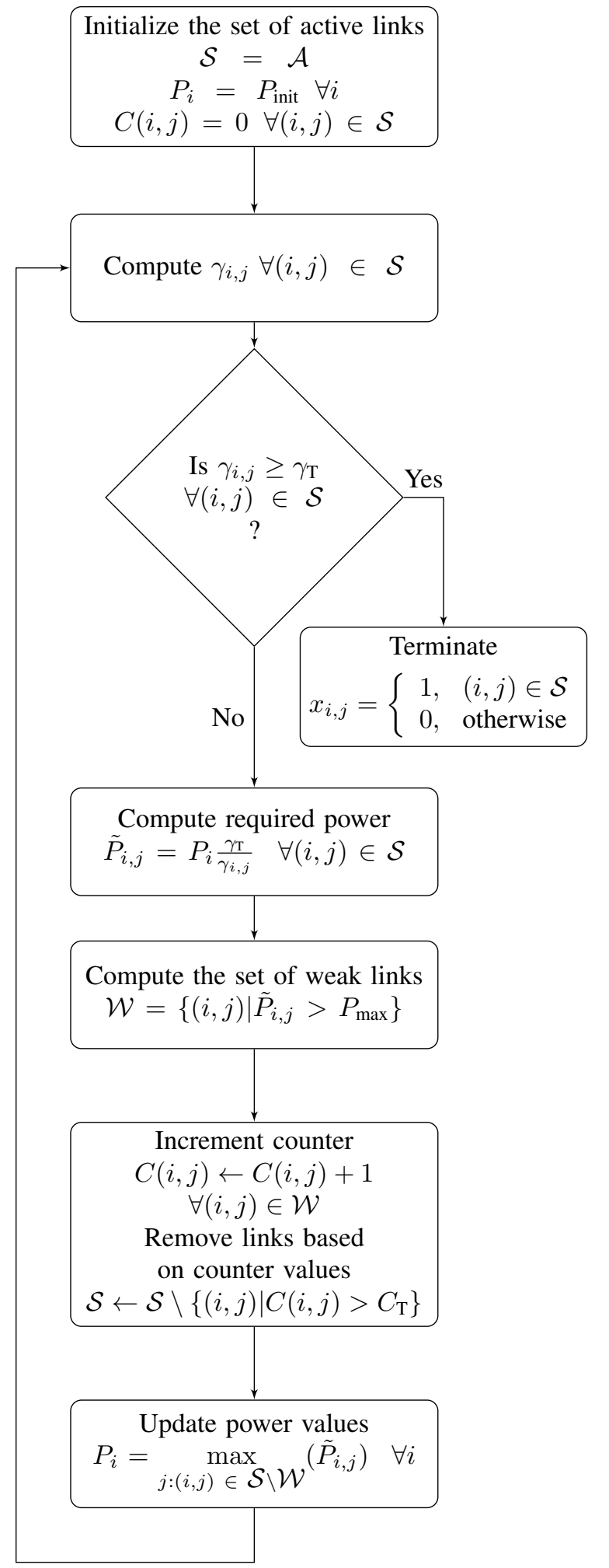

Fig. 4. Flowchart of the proposed heuristic power control algorithm

whole process described above. Finally, the algorithm will terminate when we have $\gamma_{i, j} \geq \gamma_{\mathrm{T}}$ for all $(i, j) \in \mathcal{S}$.
TABLE I

SySTEM Simulation PaRAMETERS

\begin{tabular}{|l|l|}
\hline Parameter & Value \\
\hline$P_{\max }$ & $24 \mathrm{dBm}$ \\
$P_{\text {init }}$ & $P_{\max } / 10$ \\
$\mathrm{PL}_{0}$ & $63.3 \mathrm{~dB}$ \\
$n$ & 1.77 \\
$d_{0}$ & $10 \mathrm{~m}$ \\
$\sigma_{1}$ & $3.1 \mathrm{~dB}$ \\
Penetration Loss & $10 \mathrm{~dB}$ per obstructing VUE \\
$\sigma^{2}$ & $-95.2 \mathrm{dBm}$ \\
$d_{\text {avg }}$ & $20 \mathrm{~m}$ \\
$d_{\min }$ & $10 \mathrm{~m}$ \\
$\beta$ & $1 /\left(N P_{\max }\right)$ \\
$\eta$ & $\gamma_{\mathrm{T}}\left(N P_{\max }+\sigma^{2}\right)$ \\
$C_{T}$ & 100 \\
\hline
\end{tabular}

\section{Simulation Results}

We assume an all-to-all broadcast scenario, i.e., each VUE transmits messages to all the other VUEs. This way, the set of all the intended links $\mathcal{A}$ becomes

$$
\mathcal{A}=\{(i, j): 1 \leq i \leq N, 1 \leq j \leq N, j \neq i\},
$$

with $|\mathcal{A}|=N(N-1)$. Also, to avoid CCI, we assign nonoverlapping RBs to the $N$ VUEs as mentioned above.

Moreover, we adopt the channel model of V2V links based on the highway scenario given in [15]. Specifically, the path loss in $\mathrm{dB}$ for a distance $d$ is computed as

$$
\mathrm{PL}(d)=\mathrm{PL}_{0}+10 n \log _{10}\left(d / d_{0}\right)+X_{\sigma_{1}}
$$

where $n$ is the path loss exponent, $\mathrm{PL}_{0}$ is the path loss at a reference distance $d_{0}$, and $X_{\sigma_{1}}$ represents the shadowing effect which is modeled as a zero-mean normally distributed random variable with standard deviation $\sigma_{1}$. An additional attenuation of $10 \mathrm{~dB}$ is added as penetration loss for each obstructing VUE [16], however we ignore small scale fading. The simulation parameters are summarized in Table I.

For performance evaluation, we compare our proposed two power control algorithms, i.e., the near-optimal by $\mathrm{BnB}$ and the heuristic, with the following baseline methods.

1) The equal power allocation method, where all the VUEs broadcast with the same transmit power $P_{\max }$.

2) The power control scheme proposed in [8], where each VUE updates its transmit power based on the interference function reported back from its receivers and a receiver is removed immediately as soon as its measured interference function is larger than $P_{\max }$.

For simulating the near-optimal power control solution by branch and bound method, we used Gurobi toolbox v6.0.5 [17], where we set the tolerance value for the branch and bound method as $10^{-9}$. Moreover, we set the maximum number of iteration as $10^{6}$.

We first consider a simple scenario with 3 VUEs as depicted in Fig. 5. Let $d_{i, j}$ be the distance between the $i^{\text {th }}$ VUE and $j^{\text {th }}$ VUE. The average number of successful links per VUE is evaluated in terms of $d_{1,2}$ and $d_{2,3}$, for the case $\gamma_{\mathrm{T}}=5 \mathrm{~dB}$ in Fig. 6 and the case $\gamma_{\mathrm{T}}=20 \mathrm{~dB}$ in Fig. 7. Firstly, as revealed in both Fig. 6 and Fig. 7, equal power allocation yields obviously worse performance compared to the other three methods. This illustrates the importance of power control 


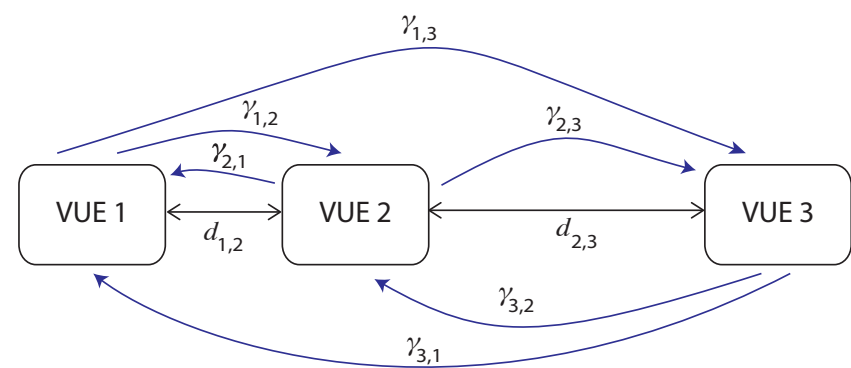

Fig. 5. System model

in broadcast $\mathrm{V} 2 \mathrm{~V}$ communications. Moreover, comparing to the scheme proposed in [8], our proposed two algorithms not only achieve a higher number of successful links but also show better robustness to a wider range of $d_{1,2}$ and $d_{2,3}$. Last but not least, the proposed near-optimal by $\mathrm{BnB}$ solution outperforms the proposed heuristic scheme at the expense of increased complexity. It is also observed that the average number of successful links is highest when $d_{1,2}=d_{2,3}$. This is due to the fact that the ACI is minimum compared to signal power, when both the intended transmitter and interferer are at the same distance from receiver.

Next we move to a general setup with $N$ VUEs dropped in a convoy as illustrated in Fig. 2. The distance between any two adjacent VUEs $z$ follows a shifted exponential distribution, with the minimum distance $d_{\min }$ and average distance $d_{\text {avg }}$. The probablity density function of $z$ is given as,

$$
f(z)= \begin{cases}\left(1 /\left(d_{\text {avg }}-d_{\text {min }}\right)\right) \cdot e^{-\frac{\left(z-d_{\text {min }}\right)}{\left(d_{\text {avg }}-d_{\min }\right)}}, & z \geq d_{\text {min }} \\ 0, & \text { otherwise }\end{cases}
$$

The average number of successful links per VUE is plotted in Fig. 8 with respect to different $N$. With increased $N$, the average number of successful links also goes up due to the increased number of the intended receivers. However, for a relatively high $N$, e.g., $N \geq 16$, the performance increases quite slowly and will eventually be flat. This is reasonable since a VUE will only be able to reach a limited number of receivers due to the ACI and noise, no matter how many VUEs exist in the network. When it comes to different power control schemes, again, the equal power allocation gives the worst performance. Also, the proposed two algorithms show clear advantages compared to the method presented in [8], especially for a higher $N$. Besides, it is worth to mention the significant gap between the near-optimal by $\mathrm{BnB}$ and heuristic algorithms. In fact, the gap gives the possibility of further improvement to attain a better tradeoff between performance and complexity.

Similarly, in Fig. 9, we plot the average number of successful links per VUE with respect to different $\gamma_{\mathrm{T}}$. Clearly, the number of successful links decreases when increasing $\gamma_{\mathrm{T}}$ due to the higher SINR threshold. Moreover, there is again a clear performance improvement of the proposed power control algorithms comparing with the baseline methods.

In Fig. 10, we compute the average probability of link success for the set of communication links with different index
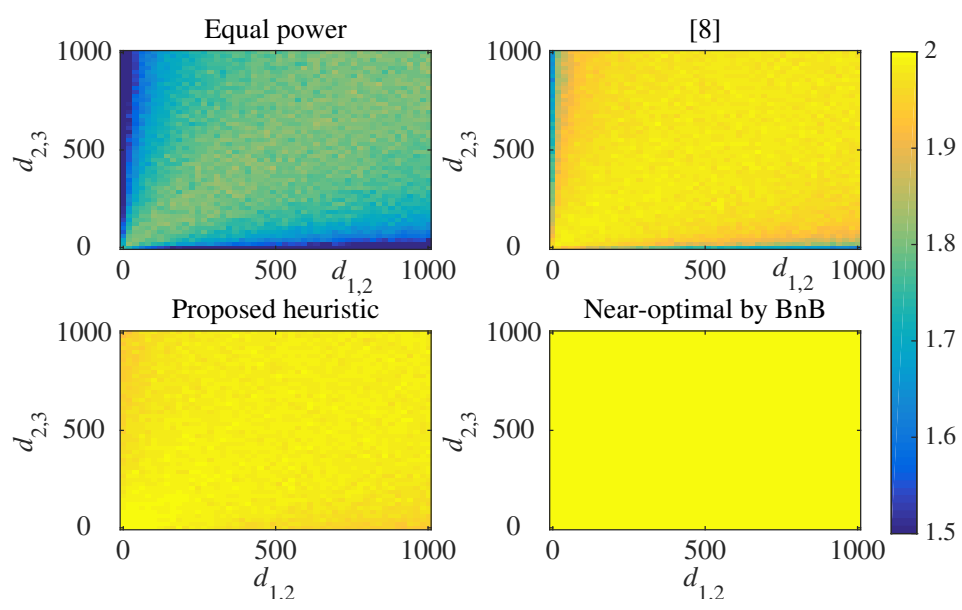

Fig. 6. Average number of successful links per VUE $\left(\gamma_{\mathrm{T}}=5 \mathrm{~dB}\right)$
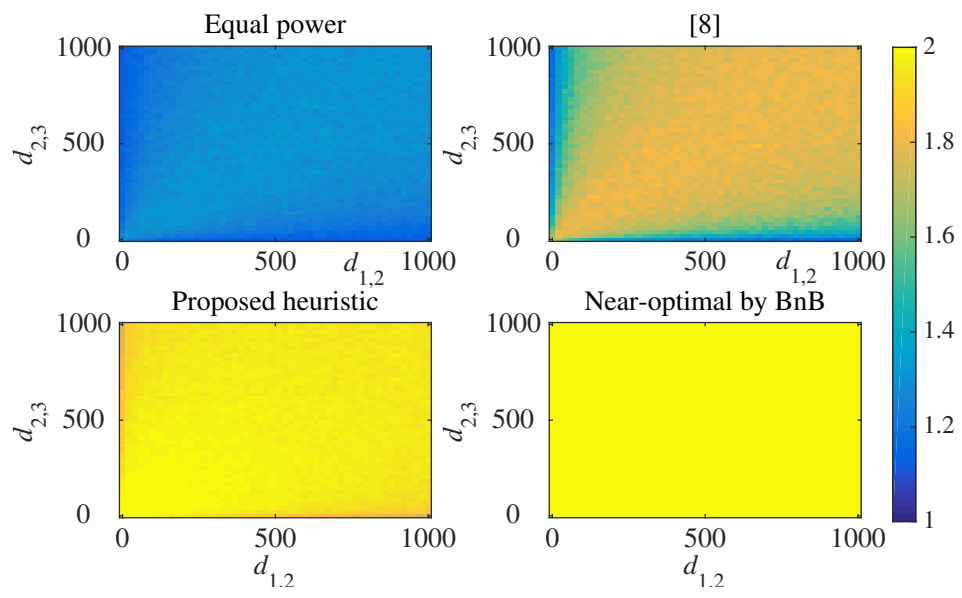

Fig. 7. Average number of successful links per VUE $\left(\gamma_{\mathrm{T}}=20 \mathrm{~dB}\right)$

distance $L$. Here we define the set with index distance $L$ as $\{(i, j):|i-j|=L\}$. As shown in Fig. 10, the nearoptimal solution by $\mathrm{BnB}$ achieves almost probability 1 for the neighboring receivers (i.e., $L=1$ ), which reveals its promising reliability performance for localized services. Moreover, both the proposed near-optimal result by $\mathrm{BnB}$ and the proposed heuristic algorithm outperform the two existing methods in all the considered neighbour indices, i.e., when $1 \leq L \leq 5$. Last but not least, we again observe a clear gap between the near optimal and the heuristic method, particularly for a smaller $L$. This observation opens the possibility of devising more sophisticated power control algorithm, yet with an acceptable complexity, to mitigate the ACI effects.

\section{CONCLUSion AND Future Work}

In this work, we studied the power control problem for broadcast V2V communications when ACI effects are taken into account. By detailed investigations, we first observed that, in the absence of CCI, the ACI has a significant impact on the reliability of $\mathrm{V} 2 \mathrm{~V}$ links. Therefore, we then formulated a power control problem to reduce the negative influence of 


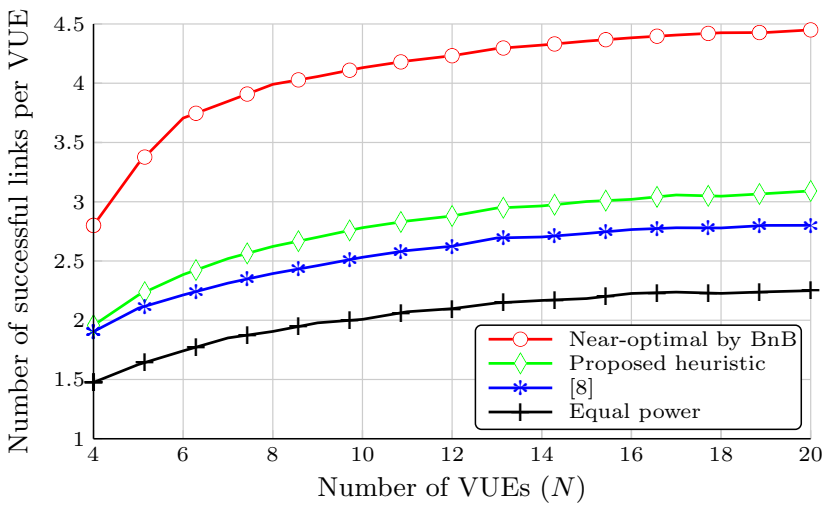

Fig. 8. Average number of successful links per VUE $\left(\gamma_{\mathrm{T}}=5 \mathrm{~dB}\right)$.

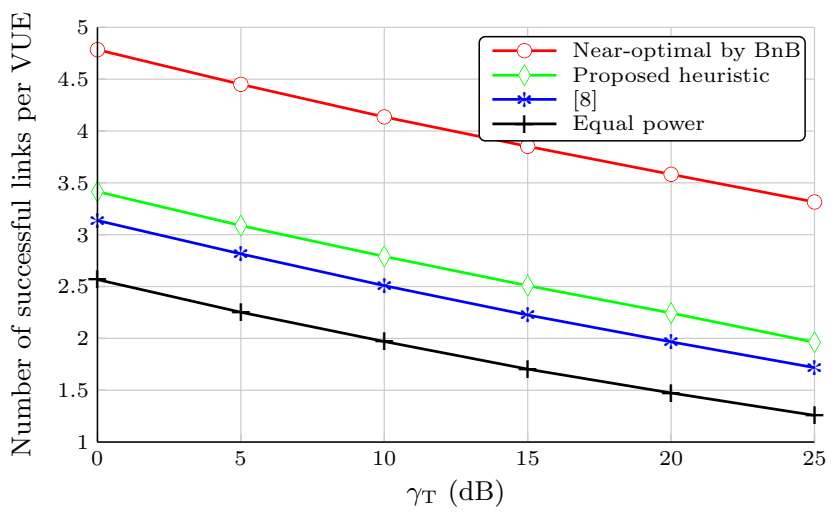

Fig. 9. Average number of successful links per VUE $(N=20)$.

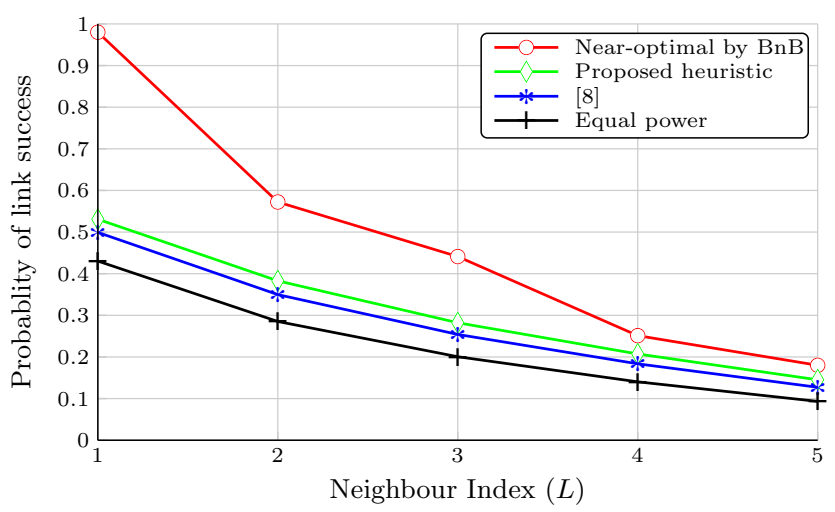

Fig. 10. Probablity of link success for various neighbour communication links $(N=20)$.

ACI. The problem was stated as an optimization problem with the main objective to maximize the number of successful V2V links, which was shown to be NP-hard. Moreover, two power control algorithms were proposed to solve the problem. The first algorithm achieves the near-optimal solution by the branch and bound method at the cost of exponentially increased worstcase complexity. The second algorithm uses a heuristic concept with much better computational efficiency at the sacrifice of performance. Finally, as illustrated by the simulation results, power control is indeed necessary when ACI exists and the proposed algorithms show obvious improvement compared to a naive equal-power scheme and the more advanced power control algorithm in [8]. However, it should be mentioned that there is a clear performance gap between the proposed near optimal and heuristic power control schemes. Future work will include formulating schemes for RB allocation and power control that achieve other tradeoffs between performance and complexity.

\section{REFERENCES}

[1] W. Sun, E. G. Ström, F. Brännström, K. Sou, and Y. Sui, "Radio resource management for D2D-based V2V communication," IEEE Transactions on Vehicular Technology, accepted for publication, 2015. [Online]. Available: http://publications.lib.chalmers.se/records/fulltext/ 222774/ \local \_222774.pdf

[2] L. Gallo and J. Härri, "Short paper: A LTE-direct broadcast mechanism for periodic vehicular safety communications," in IEEE Vehicular Networking Conference, Dec. 2013, pp. 166-169.

[3] W. L. Tan, K. Bialkowski, and M. Portmann, "Evaluating adjacent channel interference in IEEE 802.11 networks," in Proc. IEEE Vehicular Technology Conference, Spring 2010, pp. 1-5.

[4] E. Dahlman, S. Parkvall, and J. Sköld, 4G: LTE/LTE-Advanced for Mobile Broadband, 1st ed. Academic Press, 2011.

[5] H. Lu and C. Poellabauer, "Balancing broadcast reliability and transmission range in VANETs," in Proc. IEEE Vehicular Networking Conference, Dec. 2010, pp. 247-254.

[6] P. Haghani and Y.-C. Hu, "Power control for fair dynamic channel reservation in vanets," in Proc. 9th Annual IEEE Communications Society Conference on Sensor, Mesh and Ad Hoc Communications and Networks, Jun. 2012, pp. 659-667.

[7] Y. Pourmohammadi Fallah, N. Nasiriani, and H. Krishnan, "Stable and fair power control in vehicle safety networks," IEEE Transactions on Vehicular Technology, vol. PP, 2015.

[8] K. Wang, C. Chiasserini, J. Proakis, and R. Rao, "Joint scheduling and power control for multicasting in wireless ad hoc networks," in Proc. IEEE Vehicular Technology Conference, vol. 5, Oct. 2003, pp. 2915-2920.

[9] Q. Xue, A. Pantelidou, and M. Latva-Aho, "Energy-efficient scheduling and power control for multicast data," in IEEE Wireless Communications and Networking Conference, Mar. 2011, pp. 144-149.

[10] H. Soleimani and A. Boukerche, "CAMS transmission rate adaptation for vehicular safety application in LTE," in Proc. Fourth ACM International Symposium on Development and Analysis of Intelligent Vehicular Networks and Applications. New York, NY, USA: ACM, 2014, pp. 47-52. [Online]. Available: http://doi.acm.org/10.1145/ 2656346.2656347

[11] E. Yaacoub, F. Filali, and A. Abu-Dayya, "QoE enhancement of SVC video streaming over vehicular networks using cooperative LTE/802.11p communications," IEEE Journal of Selected Topics in Signal Processing, vol. 9, no. 1, pp. 37-49, Feb. 2015.

[12] 3GPP, "Evolved Universal Terrestrial Radio Access (E-UTRA); Radio Frequency (RF) system scenarios," 3rd Generation Partnership Project (3GPP), TR 36.942, Oct. 2014. [Online]. Available: http: //www.3gpp.org/ftp/Specs/html-info/36942.htm

[13] M. Andersin, Z. Rosberg, and J. Zander, "Gradual removals in cellular PCS with constrained power control and noise," in Proc. IEEE Personal Indoor and Mobile Radio Communications (PIMRC), vol. 1, Sep. 1995, pp. 56-60.

[14] Stephen Boyd and Jacob Mattingley, "Branch and bound methods, notes for EE364b," March 2007. [Online]. Available: https://see.stanford.edu/ materials/lsocoee364b/17-bb \_notes.pdf

[15] J. Karedal, N. Czink, A. Paier, F. Tufvesson, and A. Molisch, "Path loss modeling for vehicle-to-vehicle communications," IEEE Transactions on Vehicular Technology, vol. 60, no. 1, pp. 323-328, Jan. 2011.

[16] D. Vlastaras, T. Abbas, M. Nilsson, R. Whiton, M. Olbäck, and F. Tufvesson, "Impact of a truck as an obstacle on vehicle-to-vehicle communications in rural and highway scenarios," in Proc. IEEE 6th International Symposium on Wireless Vehicular Communications, 2014.

[17] Gurobi Optimization, Inc., "Gurobi optimizer reference manual," 2015. [Online]. Available: http://www.gurobi.com 